\title{
DISTRIBUCION DE STAURASTRUM PLANCTONICUM VAR. BULLOSUM (CHLOROPHYTA, DESMIDIACEAE) EN LA COLUMNA VERTICAL DE UN EMBALSE EUTROFICO A DIFERENTES ESCALAS TEMPORALES
}

\author{
M. Araúzo Sánchez; M. Alvarez Cobelas; A. Rubio Olmo \\ Centro de Investigaciones del Agua (C.S.I.C.). La Poveda, Arganda del Rey. Madrid.
}

Palabras clave: Time scales, hierarchical hypothesis, phytoplankton responses, environmental changes.

\begin{abstract}
DISTRIBUTION OF STAURASTRUM PLANCTONICUM VAR. BULLOSUM (CHLOROPHYTA, DESMIDIACEAE) IN THE VERTICAL COLUMN OF AN EUTROPHIC RESERVOIR AT DIFERENT TIME SCALES
\end{abstract}

Factors controlling population dynamics of Staurastrum planctonicum var. bullosum (Chlorophyta, Desmidiaceae) living in El Vellón reservoir (Madrid, Spain) have been considered at three time scales: monthly, daily and hourly.

In the year cycle the population appears to be related with water temperature, water density, «euphotic layer/mixed layen ratio, inorganic dissolved carbon, thermal stability of the water column and solar radiation (with these two latter factors having time lags of one and two months, respectively), indicating that this taxon thnves during the stagnation period though it can be found all year long in the reservoir. At daily time scales, within its exponential growing penod, the population only correlates with dissolved nitrogen, dissolved phosphorous and with «dissolved nitrogen/dissolved phosphorous» ratio, whereas at hourly time scales microclimatic changes seem to prevail on its dynamics, which is demonstrated by delays in correlation and by fact that factors influencing such dymamics are not the same on two close but weather-contrasting days.

These results support the hierarchical hypothesis of phytoplankton responses to environmental changes.

\section{INTRODUCCION}

El ambiente en el que se desarrolla el fitoplancton es un medio heterogéneo: los cambios de temperatura, radiación, hidrodinámica y disponibilidad de nutrientes han de ser considerados tanto en el tiempo como en el espacio. Las variaciones en estos factores nos llevan a reconocer diferentes escalas temporales en su dinámica, sin olvidar que en todas ellas existe un continuo de oscilaciones a corto plazo, de horas, incluso de minutos o segundos, que son en realidad la base sobre la que se asentarán los patrones de mayor escala. Harris $(1980,1985)$ lleva a cabo revisiones sobre cómo la existencia de diferentes escalas espaciales y temporales en el ambiente fitoplanctónico afecta a

Limnética 3: 179-187 (1987)

(c) Asociación Española de Limnología, Madrid. Spain la ecología de las algas. También se trata el problema de la escala en Allen (1977), Allen \& Starr (1982), Frempong (1981, 1984) y Reynolds (1984).

Aunque las desmidiáceas han sido objeto de numerosos estudios, casi todos ellos se han centrado exclusivamente en su taxonomía, siendo hasta ahora muy escasas las investigaciones referentes a la ecología de este grupo de algas (Lund, 1971).

En este trabajo se estudia la distribución del alga Staurastrum planctonicum var. bullosum (Chlorophyta, Desmidiaceae) en la columna vertical de un embalse eutrófico (El Vellón, Madrid) a tres escalas temporales diferentes: mensual, diaria y horaria. Se pretende buscar la relación entre las variaciones en la densidad de células de la población y la evolución 

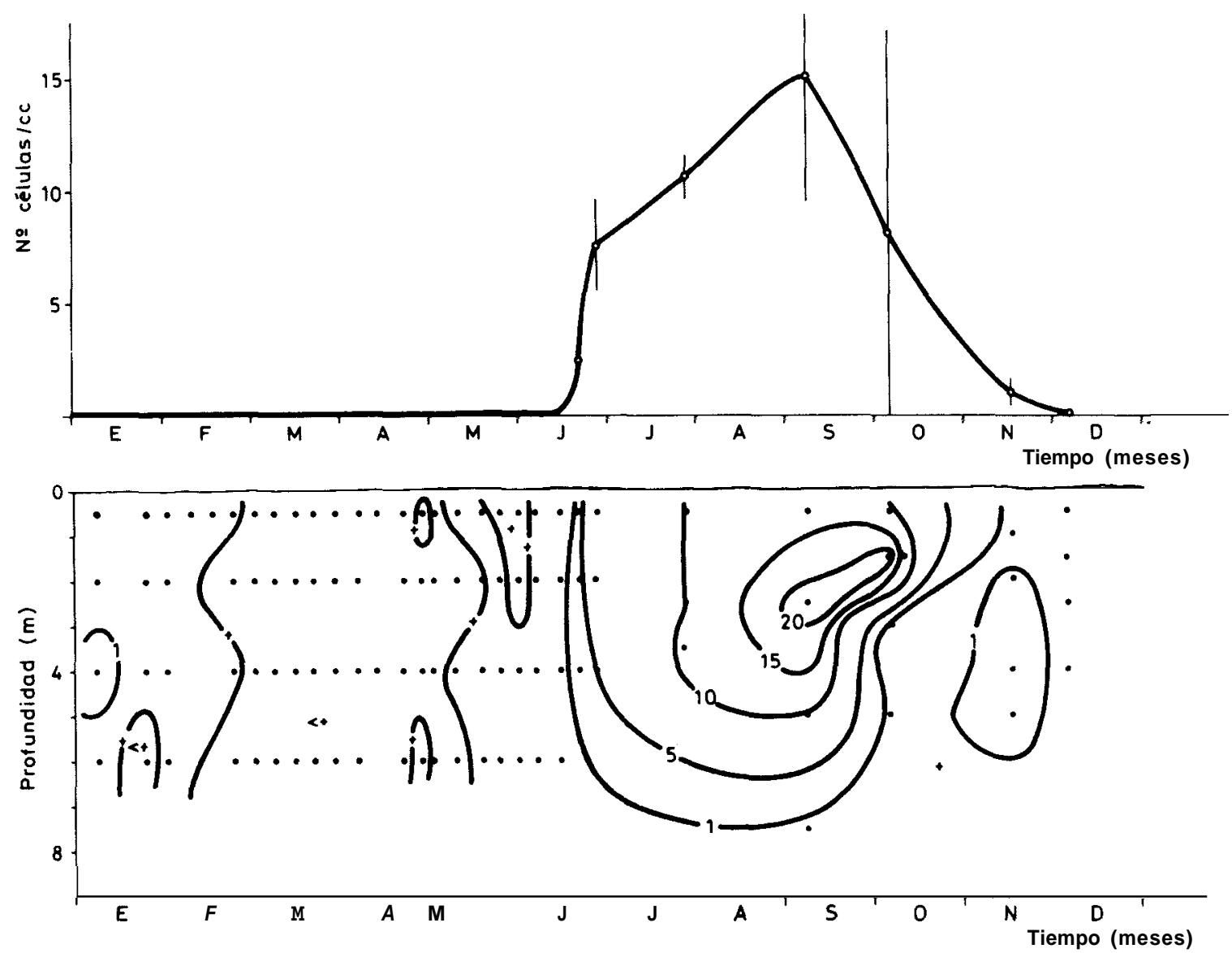

Figura 1.- Evolución temporal de la densidad de células de Staurastrum planctonicum var. bullosum en la columna vertical del embalse de El Vellón durante 1983. Barras verticales: desviacionestípicas.

Temporal evolution of cell density of Staurastrum planctonicum var. bullosum in vertical column of El Vellón reservoir in 1983. Vertical bars: standard deviations.

temporal de algunos parámetros físico-químicos durante cada uno de los periodos. Posteriormente se comparan los resultados de las tres escalas temporales en estudio.

Se han realizado trabajos sobre la ecología de esta especie a corto plazo (Arauzo et al. 1986. Alvarez \& Arauzo, datos inéditos), pero nunca se ha comparado el efecto que puedan tener las diferentes escalas temporales sobre la misma.

Las características físico-químicas y biológicas del embalse de El Vellón se describen en Margalef et al. (1977), Alvarez (1982) y Alvarez et al. (1983, 1985a, 1985b).

\section{MATERIAL Y METODOS}

Para la realización de los muestreos se eligió la es- tación más representativa de las características promedio del embalse (estación B: Alvarez et al., 1985a, 1985b).

En el transcurso del año 1983 se realizaron muestreos de carácter mensual, de enero a diciembre, diario, del 18 al 26 de junio, y durante los días 21 y 25 de dicho mes, diferentes climatológicamente entre sí, se extrajeron muestras de fitoplancton y se midieron variables físicas cada hora, desde las 7,00 hasta las 19,00 horas (hora solar). Mediante una bomba de succión se tomaron muestras de toda la columna en el periodo de mezcla (a profundidades variables), y del epilimnion $(0,5 ; 1 ; 2$ y 4 metros) en el de estratificación. El fitoplancton fue fijado con formol al $4 \%$ inmediatamente después de ser recogido. Los parámetros físico-químicos medidos fueron los siguientes: temperatura (mediante un termistor YSI-57), velocidad de la corriente a un metro de profundidad (segun 

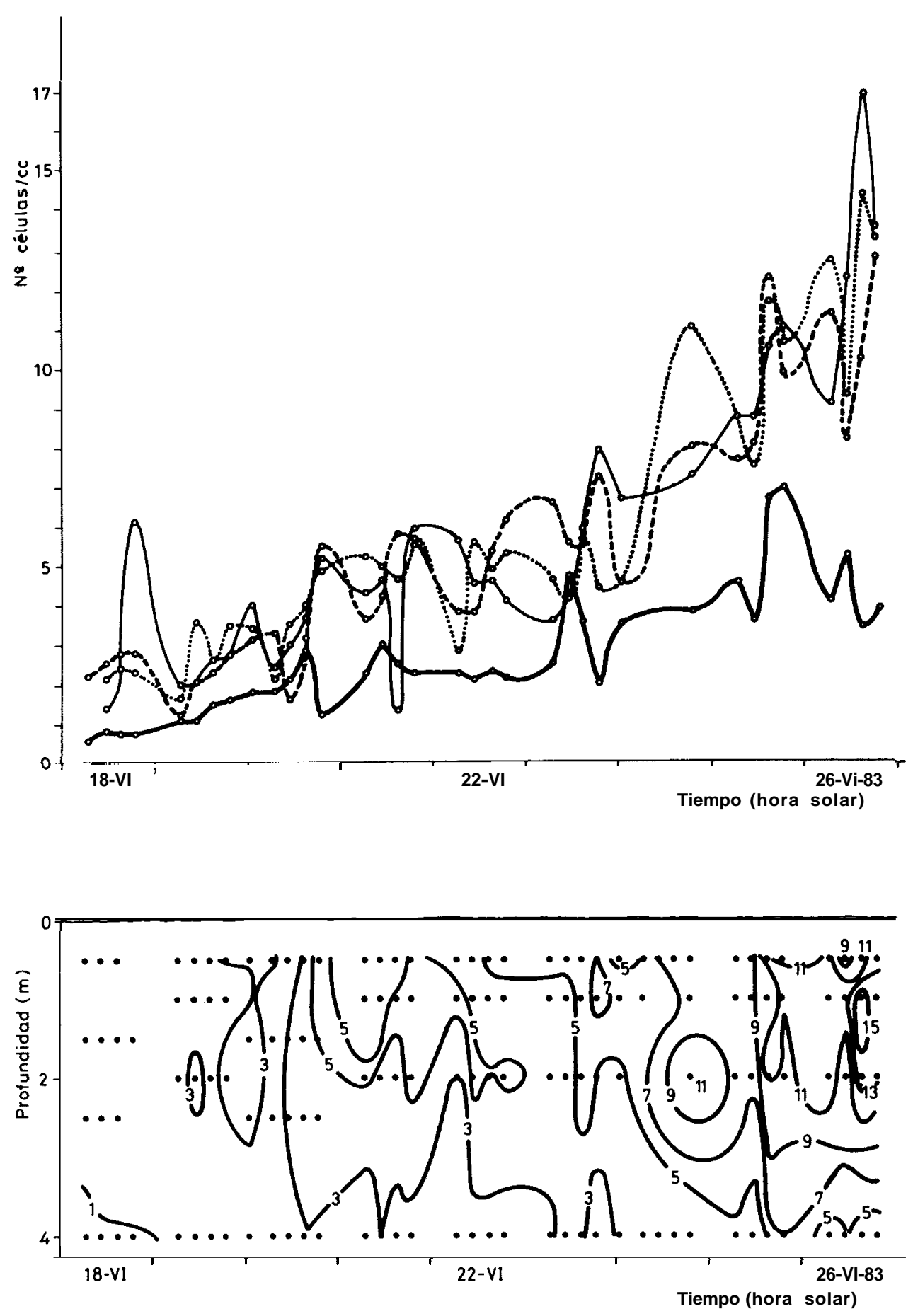

Figura 2.- Evolución temporal de la densidad de células de Staurastrum planctonicum var. bullosum en la columna vertical del embalse de El Vellón durante el periodo del 18 al 26 de junio de 1983. (-): 4 metros, ( $\cdots \cdots)$ : 2 metros, (-): 1 metro, (--): 0,5 metros.

Temporal evolution of cell density of Staurastrum planctonicumvar. bullosum in vertical column of El Vellón reservoir during the period 18th to 26th June 1983. (-): 4 m., (…): 2 m., (-): 1 m., (--): 0.5 m. 

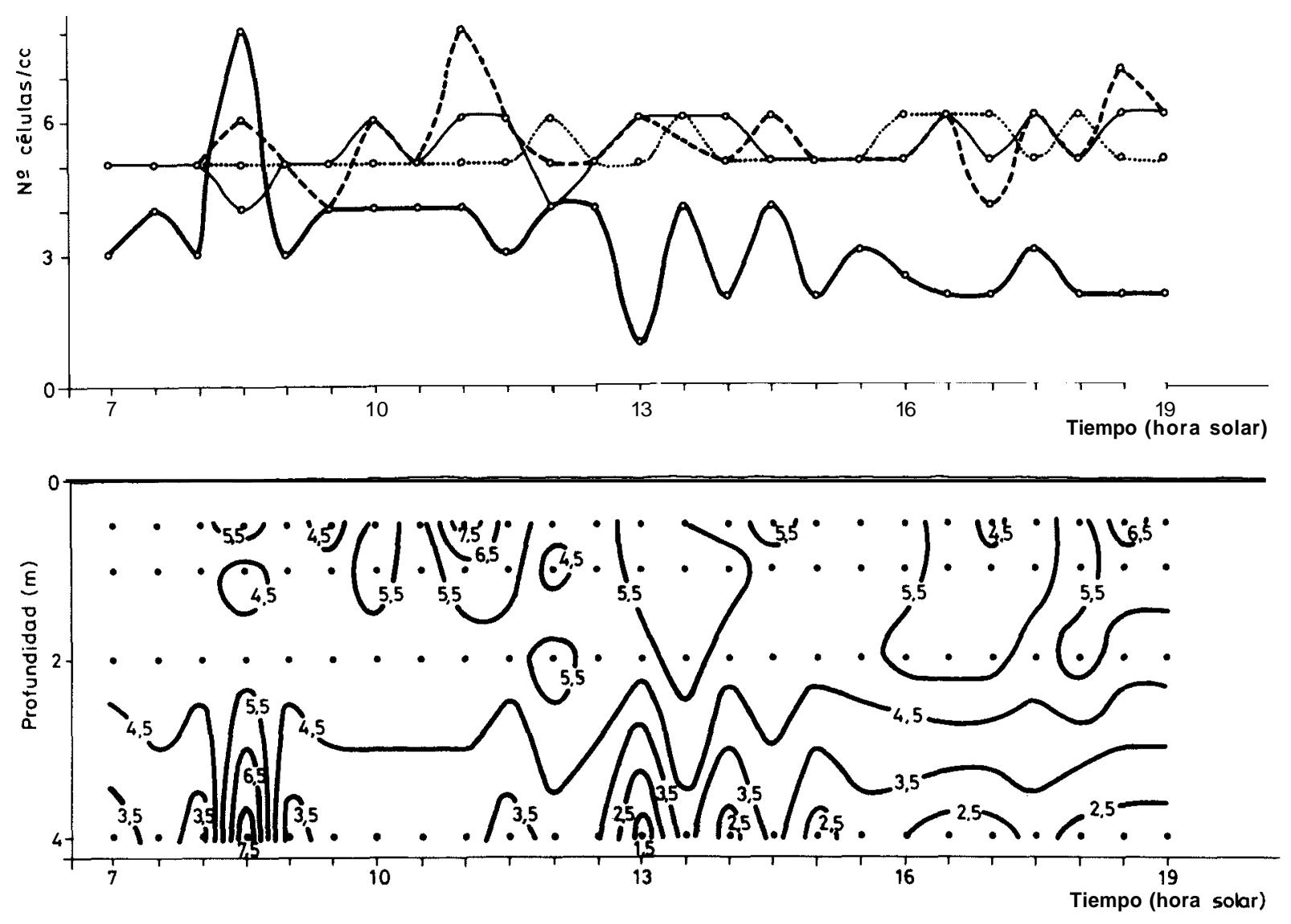

Figura 3.- Evolución temporal de la densidad de células de Staurastrum planctonicum var. bullosum en la columna vertical del embalse de El Vellón durante el día 21 de junio de 1983. Trama de las curvas igual que en figura 2.

Temporal evolution of cell density of Staurastrum planctonicum var. bullosum in vertical column of El Vellón reservoir during 21 th June 1983. Curves plotting like figure 2.

el método del Hrbacek \& Straskraba, 1966), espesor de la zona fótica (corrección del valor del disco de Secchi multiplicándolo por el coeficiente 2,7: Talling, 1971), pH (mediante un potenciómetro Orion), nitrógeno disuelto $\left(\mathrm{NH}_{4}^{+}, \mathrm{NO}_{3}^{-}, \mathrm{NO}\right.$;), fósforo reactivo soluble y carbono inorgáníco disuelto $(\mathrm{CO}, \mathrm{HCO}$, $\mathrm{CO}_{3}^{-}$) (siguiendo las técnicas analíticas del Centro de Investigaciones del Agua). La radiación promedio para cada mes se obtuvo de la publicación «Radiación solar sobre superficies inclinadas)), editada por el Centro de Estudios de la Energía (Ministerio de Industria y Energía, 1981). La densidad del agua se calculó a partir de la temperatura (Hodgman, 1947).

A partir de los datos precedentes se hallaron los cocientes «nitrógeno/fósforo» en la capa de mezcla y ((espesor de la capa iluminada/espesor de la capa de mezcla» (consideramos capa de mezcla aquella en la que el gradiente de temperatura es inferior a $1^{\circ} \mathrm{C}$ por cada metro de profundidad). Como indicadores de los procesos de advección y sedimentación se utilizaron la velocidad de la corriente a un metro y el cociente ((densidad de células a cuatro metros/densidad de células en el epilimnion», respectivamente. La frecuencia de Brunt-Vassala (Mortimer, 1974) se usó como un indicador de la estabilidad térmica vertical.

Los recuentos del alga se realizaron con un microscopio invertido siguiendo las recomendaciones de Rott (1981). Salvo en los casos de simples presencias, se contaron cien individuos en un volumen conocido de cada muestra, lo que condujo a un error en el recuento de $\pm 20 \%$ (Margalef, 1983). 

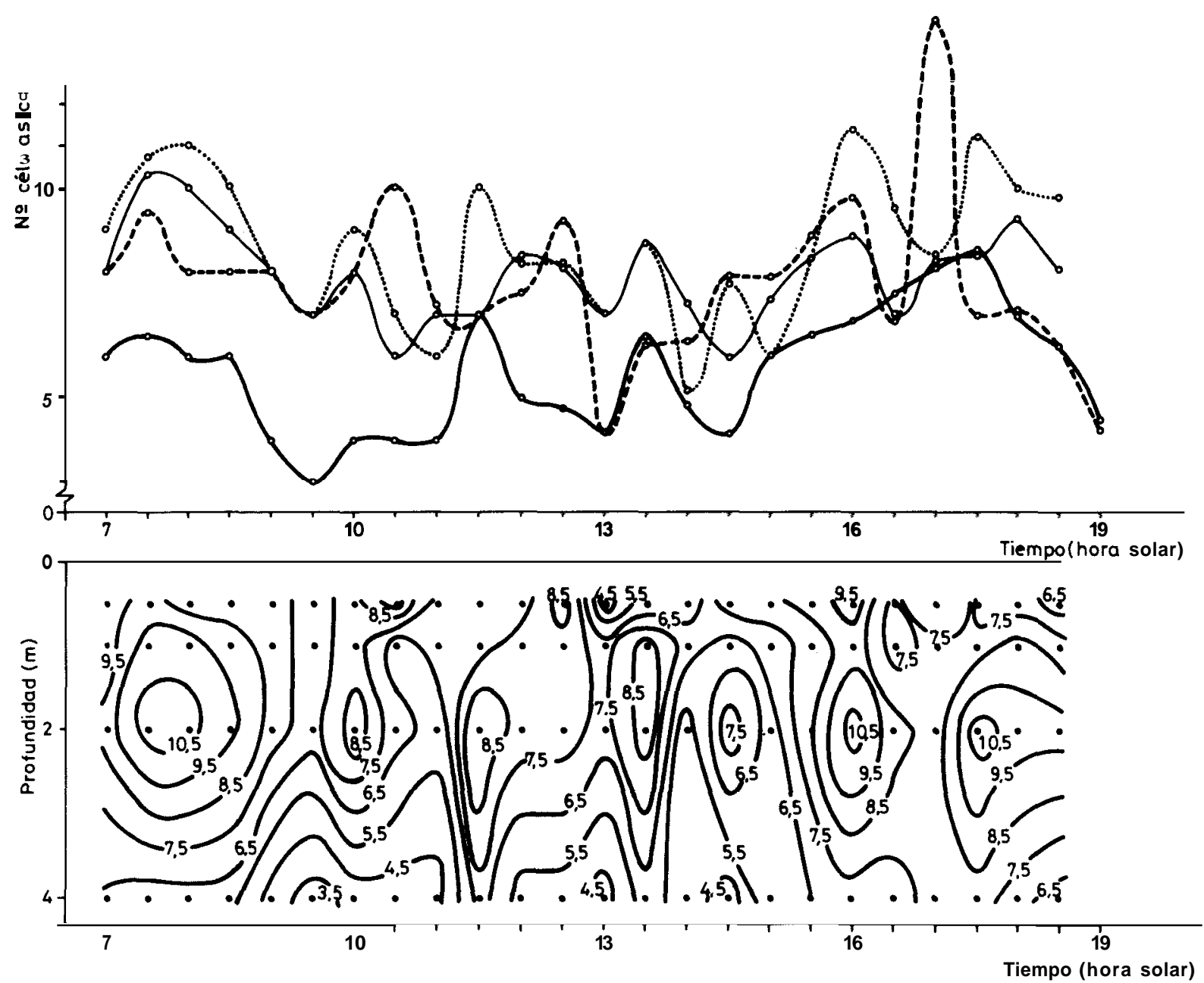

Figura 4.- Evolución temporal de la densidad de células de Staurastrum planctonicum var. bullosum en la columna vertical del embalse de El Vellón durante el día 25 de junio de 1983. Trama de las curvas igual que en figura 2.

Temporal evolution of cell density of Staurastrum planctonicum var. bullosum in vertical column of El Vellón reservoir during 25th June 1983. Curves plotting like figure 2.

Las tasas de crecimiento se obtuvieron a partir de la fórmula del modelo exponencial para el periodo del 20 al 26 de junio y de la fórmula del modelo logístico para el periodo del 26 de junio al 8 de septiembre. También se calcularon los tiempos de duplicación en ambos periodos (Guillard, 1973).

Para la escala mensual se correlacionó la densidad de células $(\mathrm{N})$ con las siguientes variables (en todos los casos se utilizan los valores promedio para la capa de mezcla): temperatura (T), densidad del agua (Da), fósforo reactivo soluble (PRS), nitrógeno disuelto $(\mathrm{Nd})$, carbono inorganico disuelto $(\mathrm{Cd})$, cociente «nitrógeno/fósforo« ( $\mathrm{N} / \mathrm{P})$, frecuencia de Brunt-Vassala $\left(\mathrm{N}^{\prime}\right)$, espesor de la capa iluminada (Zlu), cociente «espesor de la capa iluminada/espesor de la capa de la mezcla» $(\mathrm{Zlu} / \mathrm{Zm})$, radiación solar $(\mathrm{R})$, y $\mathrm{pH}$.

A escala diaria se correlacionó la densidad de células con las variables: temperatura, densidad del agua, fósforo reactivo soluble, nitrógeno disuelto, carbono inorgánico disuelto, cociente «nitrógeno/fósforo», frecuencia de Brunt-Vassala, espesor de la capa iluminada, $\mathrm{pH}$, indicador de advección (IA), e indicador de sedimentación (IS); a esta escala también se correlacionaron la densidad de células a un metro de profundidad frente al indicador de advección, y el indicador de sedimentación frente a la densidad del agua, la frecuencia de Brunt-Vassala y el espesor de la capa iluminada (en todos los casos valores promedio para el epilimnion). 
En los muestreos desarrollados cada hora, se correlacionaron los mismos parámetros que en la escala anteriormente citada, con la excepción del fósforo reactivo soluble y el cociente «nitrógeno/fósforo», de los que se carecía de datos con esta frecuencia. En ambas escalas prescindimos de los valores del cociente ((espesor de la capa iluminada/espesor de la capa de mezcla», ya que resultan redundantes con los de espesor de la capa iluminada (la capa de mezcla es siempre de cuatro metros durante este periodo).

\section{RESULTADOS}

A partir de los muestreos de carácter mensual se observa que Staurastrum planctonicum var. hullosum aparece durante todo el año en la columna vertical del embalse (fig.1), aunque en ocasiones su concentración sea muy baja. Su mayor desarrollo lo adquiere en el periodo de estratificación, en la zona del epilimnion -los muestreos por debajo de la termoclina, situada a cuatro metros de profundidad, no revelaron cantidades significativas de esta desmidiácea-. En esta época las especies fitoplanctónicas más abundantes fueron: Korschikoviella limnetica, Korschikoviella gracilipes, Oocystis horgei, Fotterella tetrachlorelloides, Coelastrum microporum, Pediastrum duplex, Planktosphaerea gelatinosa, Scenedesmus arcuatus y Phacotus lenticularis -todas ellas pertenecientes a la clase Chlorophyta-. Los máximos de densidad de células en Staurastrum parecen darse a primeros del mes de septiembre, entre 1,5 y 2,5 metros de profundidad (fig. 1, abajo). A esta escala se observa que el número de células está relacionado con los siguientes parámetros: temperatura, densidad del agua, estabilidad térmica de la columna (con desfase de un mes), radiación (con desfase de dos meses), carbono inorgánico disuelto y cociente ((espesorde la capa ilumina$\mathrm{da} /$ espesor de la capa de mezcla» (Tabla 1).

Se han calculado las tasas de crecimiento para los periodos del 20 al 26 de junio y del 26 de junio al 8 de septiembre, obteniéndose unos valores de 0,44 días - 1 para el primero y de 0,05 días -1 para el segundo, con unos tiempos de duplicación de 1,57 días y 13,80 dias, respectivamente.

Durante los muestreos diarios (18 al 26 de junio), la población se encontraba en el periodo de crecimiento exponencial (fig. 1). Los máximos en la densidad de células se localizaron en la mitad superior del epilimnion, observándose un aumento progresivo a lo largo del día a 0,5, 1 y 2 metros, hecho menos perceptible a 4 metros de profundidad (fig.2, arriba). Las correlaciones entre la densidad de células y las variables físico-químicas sólo ofrecen valores significativos para el fósforo reactivo soluble, el nitrógeno disuelto y el cociente «nitrógeno/fósforo». También se da una correlación negativa significativa entre el indicador de sedimentación y la profundidad de la capa iluminada (con desfase de un día) (tabla I).

Los días 21 y 25 (muestreados cada hora). pertenecientes así mismo al periodo exponencial de crecimiento, en apariencia no presentan grandes variaciones en la densidad de células, aunque el 25 sí se aprecia un cierto aumento al final de la tarde (figs.3 y 4). Los máximos en la densidad de células se dan en la mitad superior del epilimnion. A esta escala adquieren una mayor importancia, en cuanto a influencia en el número de células en la columna se refiere, aquellas variables físicas cuyas oscilaciones están determinadas por cambios de tipo microclimático (viento, nubes, etc.), que ocasionan ligeras y rápidas variaciones de temperatura, densidad del agua, estabilidad térmica, espesor de la zona fótica y transporte horizontal y vertical en el epilimnion (tabla I).

\section{DISCUSION}

La atención a los problemas de la escala resulta fundamental para entender la dinámica espaciotemporal del fitoplancton. Aunque se alude con frecuencia a este tipo de problemas (Haury et al., 1978), los estudios donde se aborda en poblaciones específicas naturales son muy escasos. Intuitivamente puede aceptarse que los factores determinantes del crecimiento de una población fitoplanctónica en un ecosistema concreto varían a lo largo del ciclo anual; si la especie es poco resistente a los cambios, la población desaparece: por el contrario, si los tolera, aunque adaptándose, puede seguir residiendo allí. Las adaptaciones del fitoplancton para vivir en ambientes tan inestables son muy variadas (Kilham \& Kilham, 1981) y en buena medida está aún por descubrir.

En el caso concreto de las desmidiáceas del plancton, los estudios de campo sobre sus poblaciones revelan que se trata de especies que crecen preferentemente durante la época estival (Reynolds, 1940; Duthie, 1965; Lund, 1971; Margalef et al., 1977). Staurastrum planctonicum var. hullosum también sigue esta tendencia, desarrollándose fundamentalmente durante el verano. Sin embargo, pueden encontrarse células de esta especie en la columna vertical del embalse de El Vellón durante todo el año, lo cual indica que nos encontramos ante una especie euplanctónica (Hutchinson, 1967). No se han observado zigotos. Lo más evidente de este estudio es que la población responde a distintos factores ambientales según la escala temporal elegida. Así, a escala mensual, el desarrollo del alga parece estar relacionado con parámetros que varían de modo estacional, tales como la temperatura, la densidad del agua, el cociente «espesor de la capa iluminada/espesor de la capa de mezcla», la ra- 
MENSUAL

$N: T$

0,69 *

ns

$\mathrm{N}$ : PRS

0,68 *

ns

$\mathrm{N}: \mathrm{N}_{\mathrm{d}}$

$\mathrm{N}: \mathrm{N} / \mathrm{P}$

$\mathrm{ns}$

$0,73^{*}$

$\mathrm{N}: \mathrm{N}^{2}$

$\mathrm{N}: \mathrm{Zlu}$

$\mathrm{N}: \mathrm{Zlu} / \mathrm{Zm}$

$\mathrm{N}: \mathrm{R}$

$\mathrm{N}: \mathrm{pH}$

$N: I A$

$\mathrm{N}: \mathrm{IS}$

$\mathrm{N}_{1 \mathrm{~m}}: \mathrm{IA}$

IS : D a

$I S: N^{2}$

IS : $\mathrm{Z} 1 \mathrm{u}$

ns
$-0,69$ *

$\mathrm{N}$ desf. 1 mes

$\mathrm{ns}$

$\mathrm{ns}$

$\mathrm{ns}$

$\mathrm{ns}$

$0,61 *$

$0,75 *$

$\mathrm{N}$ desf. 2 meses

ns

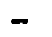

ns $\mathrm{ns}$

DIARIA

HORARIA

$21-\mathrm{VI}-83$

HORARIA

$25-\mathrm{VI}-83$

$-0,76 * * \quad 0,67 *$

$\mathrm{N}$ desf. 2 horas $\mathrm{N}$ desf. 2 horas

$0,78 *$

$\mathrm{N}$ desf. 1 día

0,72 *

$0,76 * \star$

$\mathrm{N}$ desf. 2 horas

$-0,74^{\star}$

$\mathrm{N}$ desf. 4 horas

0,53 *

ns

ns

ns

n s

ns

$-0,90 * \star$

I S desf. 1 día

$-0,71 * *$

ns

n s

$0,68 *$

n s

$0,53^{\star}$
$\mathrm{N}$ desf. 2 horas

$-0,65 *$

$\mathrm{N}$ desf. 1 hora

$\mathrm{N}$ desf. 2 horas

$-$

-

ns ns

$0,60+$

$\mathrm{N}$ desf. 2 horas

ns

$0,67 *$

$\mathrm{N}_{1 \mathrm{~m}}$ desf. 2 horas

$-0,82 \star \star$

$-0,69+$

I $S$ desf. 3 horas

$-0,58+$

IS desf. 2 horas

Tabla 1.- Coeficientes de correlación log-log significativos para las tres escalas temporales en estudio. Valores promedio para la capa de mezcla. Todos los datos han sido también correlacionados con desfases temporales (indicado en la tabla como «desf.»). Para los símbolos véase material y métodos. $* *$ : significativo para $p \leqslant 0,01 . *$ : significativo para $p \leqslant 0,05 .+$ : significativo para $p \leqslant 0,10$. ns: no significativo estadísticamente.

Significant $\log -\log$ correlation coeffitients for the different temporal scales. Average values for the mix layer. All data has been tested with temporal lags («desf.»). For symbols see matenal and methods. **: significant at $p \leqslant 0,01 . *$ : significant at $p \leqslant 0,05$. $+:$ significant at $\mathrm{p} \leqslant 0,10$, ns: no signification. 
diación solar y la estabilidad térmica de la columna vertical, aunque en estos Últimos factores con un cierto desfase temporal. Este hecho es similar al referido por Lund (1971) para las desmidiáceas de Windermere. Las tasas de crecimiento una vez comenzada la fase exponencial, varían a lo largo del verano, fenómeno que también observa Lund (1971). Sin embargo, las tasas de crecimiento son siempre inferiores a las referidas por dicho autor para otra especie del mismo género, Staurastrum lunatum.

A una escala de tiempo más breve (diaria), y durante el transcurso de la fase exponencial de crecimiento del alga, la población parece relacionarse con parámetros estrictamente nutricionales, tales como las variaciones en la concentración de fósforo reactivo soluble y nitrógeno disuelto (con desfase de un día), así como del cociente «nitrogéno/fósforo», que aumenta gradualmente por el creciente consumo de fósforo ocasionado por la comunidad fitoplanctónica, de la que forma parte Staurastrum.

Harris (1983) señala que las adaptaciones de una población de algas a la variación ambiental se produce con retardos proporcionales al tiempo de duplicación de la población. A pesar de todo, no se han observado respuestas a la variación ambiental con retardos temporales similares a su tiempo de duplicación (casi de dos días). La ausencia de una respuesta de este tipo podría deberse a que el cálculo de las tasas de crecimiento y los tiempos de duplicación no se basa en la consideración de procesos estrictamente reproductivos, sino que se hallan distorsionados por los efectos del transporte horizontal y vertical en la columna de agua (Arauzo et al., 1986). No ha sido posible evaluar la tasa de sedimentación ya que se carecía de datos referentes al plancton del hipolimnion.

La dinámica por horas parece estar más condicionada por procesos de tipo microclimático, prueba de lo cual es que todas las correlaciones significativas lo han sido con desfase, además de no ser siempre las mismas en dias próximos pero diferentes climatológicamente.

Por otra parte, hay que señalar que existen algunos parámetros muy relacionados entre sí, como la temperatura, la densidad del agua y la estabilidad térmica de la columna, que deben ser interpretados de forma distinta con arreglo a la escala temporal de trabajo: a escala mensual son mayores sus variaciones, hasta tal punto que condicionan la existencia de periodos de mezcla y de estratificación térmica a lo largo del año, que a su vez contribuye de forma decisiva en la selección de las especies fitoplanctónicas predominantes en cada periodo. A escalas temporales pequeñas las variaciones de estos parámetros son de menor magnitud, de modo que no son suficientes para romper la estructura térmica de la masa acuática, pero sí de condicionar la dinámica del epilimnion: las rápidas variaciones en los procesos de transporte horizontal y vertical, contribuyen a la modificación de los patrones de distribución de las algas.

Los resultados de este estudio abonan experimentalmente la hipótesis de Harris (1980) sobre la jerarquía de las respuestas del fitoplancton al ambiente: según la escala considerada, el fitoplancton responde a unos factores o a otros, que van desde procesos estacionales (radiación, temperatura, etc.), hasta los de minutos o segundos (oscilaciones microclimáticas: variación en la velocidad del viento, presencia de nubes, etc.). En caso de que la población no pudiera adaptarse a estos procesos, desaparecería, pero al menos en especies grandes como Staurastrum planctonicum var. bullosum esto no parece suceder, debido a que es capaz de explotar con bastante éxito la inestabilidad ambiental.

\section{BIBLIOCRAFIA}

Allen, T.F.H. 1977. Scale in microscopic algal ecology: a neglected dimension. Phycologia, 16:253-257.

Allen, T.H.F. \& Starr, T.B. 1982. Hierarchy. Perspectives for ecological complexity. University of Chicago. U.S.A. 310 págs.

Alvarez, M. 1982. Una limnologia de la cuenca del embalse de El Vellón (Madrid). Tesis doctoral. Univ. Complutense. Madrid. 683 pp.

Alvarez, M.; Velasco, J.L.; Rubio, A.; Colmenarejo, J.M. 1983. Las tendencias a la eutrofización en el embalse de El Vellón (Madrid). Actas V Cong. Nal. Química. 3:565-574.

Alvarez, M.; Velasco, J.L.; Rubio, A.; Acosta, F.J. 1985a. A limnology of El Vellón reservoir in 1980 and 1981. 1. Some physicochemical conditions, with special reference to horizontal patterns. Stud. Oecol. (en prensa).

Alvarez, M.; Velasco, J.L.; Rubio, A.; Acosta, F.J. 1985b. A limnology of El Vellon reservoir in 1980 and 1981. II. Phytoplankton dynamics. Stud. Oecol. (en prensa).

Arauzo, M.; Alvarez, M.; Rubio, A. 1986. Crecimiento y citocinesis de Staurastrum planktonicum var. bullosum Teiling (Zygophyceae, Desmidiaceae) en un embalse eutrófico. Limnética. (en prensa).

Centro de Estudios de la Energía. 1981. Radiación solar sobre superficies inclinadas. Ministerio de Industria y Energía. Madrid. 323 págs.

Duthie, H.C. 1965. Some observations on ecology of Desmids. J. Ecol. 53:695-703

Frempong, E. 1981. Diel variation in thc abundance, vertical distribution, and species composition of phytoplankton in an eutrophic English lake. J. Ecol. 69:919-939.

Frempong, E. 1984. A seasonal sequence of diel distribution patterns for the planktonic dinoflagellate Ceratitum hirundinella in an eutrophic lake. Freshwat. Biol., 14:401-421.

Guillard, R.R.L. 1973. Division rates. In: Hand book of phy- 
cological methods. (Stein, J.R. ed.). Cambridge University Press. London. 448 pags.

Harris, G.P. 1980. Temporal and spatial scales in phytoplankton ecology. Mechanisms, methods, models and management. Can. J. Fish. Aquat. Sci., 37: 877-900.

Harris, G.P. 1983. Mixed layer physic and phytoplankton populations: Studies in equilibrium and nonequilibrium ecology. Progress in Phycological Research, 2: 1-52.

Harris, G.P. 1985. The answer lies in the nesting behaviour. Freshwat. Biol., 15: 375-380.

Haury, L.R.; Mc Gowan, J.A.; Wiebe, P.H. 1978. Patterns and processes in the time-space scales of plankton distribution. In: Spatial pattern in plankton communities (Steel, J.H. ed.): 227-327. Plenum Press. New York, London. 470 págs.

Hodgman, C.D. 1947. Handbook of chemistry and physics. 30th ed. CRP Co. Ohio. 2.686 págs.

Hrbacek, J. \& Straskraba, M. 1966. Horizontal and vertical distribution of temperature, oxygen, $\mathrm{pH}$ and water movements in Slapy reservoir (1958-1960). Hydrobiol. Stud., 1: 7-40.

Hutchinson, G.E. 1967. A treatise on limnology. Vol. II. Wiley. New York, Sidney, London. 1.115 págs.
Kilham, P. \& Kilham, S.S. 1981. The evolutionary ecology of Phytoplankton. In: The physiological ecology of phytoplankton (Morris, I. ed.): 571-597. Blackwell. Oxford, 625 pags.

Lund, J.W.G. 1971. The seasonal periodicity of three planktonic desmids in Windermere. Mitt. Internat. Verein. Limnol., 19: 3-25.

Margalef, R.; Planas, M.D.; Armengol, J.; Vidal, A.; Prat, N.; Guisset, A.; Toja, J.; Estrada, M. 1977. Limnología de los embalses españoles. MOPU. $422+85$ págs.

Margalef, R. 1983. Limnologia. Omega. Barcelona. 1.010 págs.

Mortimer, C.H. 1974. Lake hydrodynamics. Mitt. Internat. Verein. Limnol., 20: 124-197.

Reynolds, N. 1940. Seasonal variations in Staurastrum paradoxum. New. Phytol., 39: 86-89.

Reynolds, C.S. 1984. The ecology of freshwater phytoplankton. Cambridge University Press. Cambridge, 384 págs.

Rott, E. 1981. Some results from phytoplankton counting intercalibrations. Schweiz. Hydrol., 43: 34-62.

Tailling, J.F. 1971. The underwater light climate as a controlling factor in the production ecology of Sreshwater phytoplankton. Mitt. Internat. Verein. Limnol., 19: 214-243. 
. 